\title{
A Fast and Efficient Coding Algorithm for HEVC System based on Texture Analysis of Entropy Difference
}

\author{
Qiuwen Zhang, Kunqiang Huang*, Xiao Wang, Yong Gan \\ College of Computer and Communication Engineering, Zhengzhou University of Light Industry, Zhengzhou 450002, China
}

\begin{abstract}
With the video display technique developing, High Efficiency Video Coding (HEVC) to optimize coding efficiency for video coder is proposed. However, the latest coding standard HEVC has a good performance. In the meantime, it introduces large computational complexity in mode decision process. In the coding process, the encoding mode of a coding block is selected in numbers candidate. Therefore, once a coding block in the sequences encoded as skip mode, coding time would be saved largely since the simplification of rate-distortion (RD) cost calculation. In this paper, a decision method of coding blocks (CBs) based on texture analysis of entropy is proposed. Even though a CB included in homogeneous regions in natural test video sequences, the entropy value between current and neighbouring reference coding blocks is computed to decide the CB could be encoded as skip mode. Early skip mode detection can omit unnecessary mode decision since it results in huge computational complexity for RD cost calculation in conventional method. After the detection process, the CBs would be encoded as skip mode directly. Extensive experiment concludes that our method has better performance for computational complexity compared to conventional coding method with a negligible loss.
\end{abstract}

Keywords: HEVC; mode decision; computational complexity; entropy difference

(Submitted on July 25, 2017; Revised on August 30, 2017; Accepted on September 15, 2017)

(This paper was presented at the Third International Symposium on System and Software Reliability.)

(C) 2017 Totem Publisher, Inc. All rights reserved.

\section{Introduction}

High Efficiency Video Coding (HEVC) [16] achieves advanced coding performance with respect to last coding standard H.264 since almost 50\% bit optimization with similar coding efficiency [12]. Moreover, with the development of information and visual augmented method, high efficiency visual systems have drawn more and more attentions for consumer and research institutes. HEVC has good performance while introducing huge computational complexity for mode decision. Hence, saving the encoding time of CBs is a difficulty issue now, according to the features of coding process. The fast algorithms for real-time application of HEVC codec have been proposed which has considerable enhancement compared with original HEVC encoder.

HEVC introduces some new tools that has multiple coding block partitioning patterns extended from coding tree unit (CTU) [10] which is different with previous standard. And, the tools are inherited from macroblock (MB) of previous H.264. Moreover, the CTU coding method has more free size selection for coding tree block (CTB). These CTBs are composed by a set of corresponding flexible and different size coding blocks (CBs). Encoder performs the RD optimized process for all potential split pattern groups of CBs to obtain the least RD cost, which represents the best cooperation. The best combination groups of CBs are encoded as video bit stream [2,11] to transmit in HEVC later.

In this paper, the detection algorithm is applied to CBs to decide skip mode or not. Generally, skip mode is the peculiar situation in CB partitioning pattern which the process of coding motion vector prediction and residual are omitted since they both are quantized to zero [6], skip mode as well as a special situation of intra prediction. Therefore, the skip mode is of

\footnotetext{
* Corresponding author.

E-mail address: huangkqiang@163.com.
} 
importance to reduce computational complexity of RD calculation in conventional coder, which omits some modes decision for numbers of candidates. The detection process in this paper utilizes entropy and entropy difference between the CBs to decide skip mode for the CB. In spite of the CB in motionless or motion regions, a CB could utilize skip mode to code if the difference of entropy value meets latter conditions or the threshold. In the motionless region of images, the constraint conditions are proposed. Meanwhile, the CBs in motion region should take the advantage of entropy to decide the skip. After these two detection procedures, numbers candidate modes are removable and skip mode is determinate. Therefore, this detection method has meaningful enlightenment to reduce computational complexity for RD calculation, which utilizes much coding time for some modes decision.

The rest of this paper is presented in the following. Some related woks are shown in section 2. The proposed method is fully introduced in section 3. The following experiment results are demonstrated in section 4. Lastly, the proposed method is concluded in section 5 .

\section{Related Work}

As the HEVC coding tools developed fast, some state-of-the-art algorithms are proposed. The fast coding unit partitioning algorithm in intra prediction process is proposed in [15], which achieves apparently saving for computational complexity with a little loss in RD cost calculation process. A fast edge detection algorithm in the preprocessing procedure of video frames is proposed for intra prediction of HEVC in [18] which contributes to reducing the computational complexity. A method that estimates the bits of angle prediction mode in CABAC process based on regression is presented in [4], which avoids performing bits computing in CABAC encoding process. A fast coding unit partitioning algorithm in [5] for HEVC encoder by utilizing joint online and offline learning makes a fast decision for coding unit partitioning process based on Bayesian decision. To end the process of block size determinate in advance, a method for size decision of intra pictures by utilizing partial image features is proposed in [14], utilizing the image itself to reduce coding time apparently. An intra prediction mode decision method in [8] utilizes the local binary patterns of an encoded block to achieve time saving. A fast blocks coding algorithm of intra coding utilizing the content feature of screen content coding is presented in [9], which ends the quadtree partition process by taking the advantage of motionless blocks partition as bigger size. Two methods of mode and block size decision are presented in [22], utilizing the gradient-based method for coding unit partition process to reduce encoding time distinctly. A low complexity texture video coding mode decision of dependent texture views is proposed in [19], achieving encoding time saving and complexity reduction. An early skip mode decision method for HEVC is proposed in [3], utilizing the texture advantages of motion or motionless region, which could reduce encoding time apparently.

The aforementioned algorithms are all developed for the time saving or computational complexity reduction in HEVC. However, most of these fast algorithms pay close attention to the process of CB encoding or CU partitioning whereas have no emphasis on the detection process of CBs in the sequence before the encoding procedure. Additionally, we should take full use of the characteristics that motion region contains large amount CBs could be encoded as skip mode. Therefore, improvement on how to further reduce mode decision calculated quantity for a detection of CBs before RD cost calculation can be done.

\section{Proposed work}

\subsection{Analysis of Skip Mode}

The CU partitioning structure of HEVC inter prediction unit has eight candidates that include four symmetric and four asymmetric partitioning modes. And, the especial partitioning structure of skip mode should be $2 N \times 2 N$ size. Moreover, the skipped mode of prediction unit could be regarded as the simplest coding mode without coding the motion vector between two CBs, which is quantized to zero. Therefore, the skip mode should meet the two occasions as below:

- Perform subtraction operations on two motion vector of the current and referenced prediction unit and the result is zero.

- $\quad$ Residuals are no need to be encoded since it equal to zero.

Table 1 reveals the probability of skipped CU in some test video sequences. The Traffic and Park Scene has an abundant motionless region. Furthermore, the BasketballPass contains more motion region while the BQTerrace has an important effect on coding frame when camera parameters change. Table 1 reveals that the minimum value $32.50 \%$ of CUs is skip mode for $8 \times 8$ and maximum value $81.37 \%$ of CUs selects skip mode for $64 \times 64$ with QP 22, 27, 32, 37. And, the average percentage of different size CU with four QPs is higher than $58 \%$. Therefore, making a decision for skip mode before perform the complicated RD costs of prediction blocks which can apparently save much coding time for HEVC original encoder. 
Table 1. Percentage of CUs encoded as skip for some sequences with four QPs

\begin{tabular}{|c|c|c|c|c|c|}
\hline \multirow{2}{*}{ sequences } & \multirow{2}{*}{ QP } & \multicolumn{4}{|c|}{ Skip mode for different CU size(\%) } \\
\hline & & $64 \times 64$ & $32 \times 32$ & $16 \times 16$ & $8 \times 8$ \\
\hline \multirow{5}{*}{$\begin{array}{c}\text { Traffic } \\
(2560 \times 1600)\end{array}$} & 22 & 79 & 60 & 46 & 35 \\
\hline & 27 & 85 & 64 & 51 & 36 \\
\hline & 32 & 89 & 68 & 52 & 33 \\
\hline & 37 & 93 & 70 & 48 & 28 \\
\hline & Avg & 86.5 & 65.50 & 49.25 & 33.00 \\
\hline \multirow{5}{*}{$\begin{array}{c}\text { Park Scene } \\
(1920 \times 1080)\end{array}$} & 22 & 70 & 64 & 45 & 30 \\
\hline & 27 & 82 & 67 & 51 & 35 \\
\hline & 32 & 88 & 69 & 54 & 37 \\
\hline & 37 & 92 & 69 & 51 & 40 \\
\hline & Avg & 83.00 & 67.25 & 50.25 & 35.50 \\
\hline \multirow{5}{*}{$\begin{array}{c}\text { BQ Terrace } \\
(1920 \times 1080)\end{array}$} & 22 & 68 & 47 & 64 & 26 \\
\hline & 27 & 82 & 68 & 51 & 39 \\
\hline & 32 & 91 & 71 & 60 & 46 \\
\hline & 37 & 94 & 74 & 66 & 50 \\
\hline & Avg & 83.75 & 65.00 & 60.25 & 40.25 \\
\hline \multirow{5}{*}{$\begin{array}{l}\text { Parry Scene } \\
(832 \times 480)\end{array}$} & 22 & 87 & 57 & 33 & 20 \\
\hline & 27 & 86 & 64 & 43 & 25 \\
\hline & 32 & 86 & 66 & 46 & 27 \\
\hline & 37 & 90 & 64 & 46 & 28 \\
\hline & Avg & 87.25 & 62.75 & 60.25 & 25.00 \\
\hline \multirow{5}{*}{$\begin{array}{l}\text { Blowing Bubbles } \\
\quad(416 \times 240)\end{array}$} & 22 & 51 & 46 & 36 & 23 \\
\hline & 27 & 56 & 53 & 42 & 28 \\
\hline & 32 & 72 & 59 & 48 & 30 \\
\hline & 37 & 75 & 62 & 54 & 28 \\
\hline & Avg & 63.50 & 55.00 & 45.00 & 27.25 \\
\hline \multirow{5}{*}{$\begin{array}{c}\text { Basketball Pass } \\
(416 \times 240)\end{array}$} & 22 & 82 & 82 & 61 & 33 \\
\hline & 27 & 84 & 83 & 66 & 36 \\
\hline & 32 & 84 & 83 & 69 & 35 \\
\hline & 37 & 87 & 85 & 72 & 32 \\
\hline & Avg & 84.25 & 83.25 & $\begin{array}{l}67.00 \\
\end{array}$ & 34.00 \\
\hline average & -- & 81.38 & 66.46 & 52.33 & 32.50 \\
\hline
\end{tabular}

\subsection{Detect the Skip Mode of motionless CBs}

The location of the referenced CUs in neighboring list 0 and list 1 are shown in Figure 1, which are extracted from all available referenced frames. The corresponding motion vectors $\mathrm{MV}_{\mathrm{L} 0}$ and $\mathrm{MV}_{\mathrm{L} 1}$ could be computed by utilizing the CUs and then start motion prediction procedures for current frame.

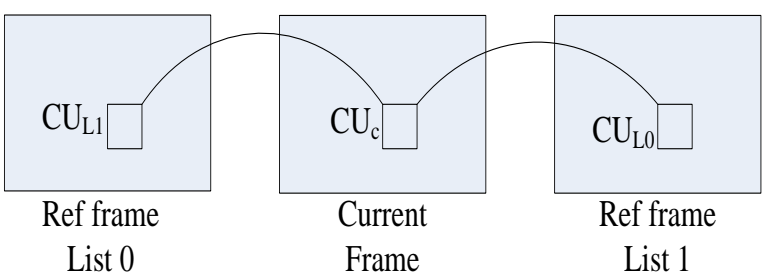

Figure 1. The skip $\mathrm{CB}$ of reference pictures

If a $\mathrm{CB}$ skips coding process, motion vector is needless to compress and residuals equal to zero. Based on these features, the significant amounts of CBs are contained in homogeneous regions of a sequence would be omitted to code [7]. Therefore, the detection of motionless regions and CUs is of importance since the frames have large stationary regions.

In this detection method, the RD computation process for $2 N \times 2 N \mathrm{CB}$ and the absolute value decision process for $\mathrm{MV}_{\mathrm{L} 0}$ and $\mathrm{MV}_{\mathrm{L} 1}$ are performed. Then, if the sum of motion vectors is zero and residuals are zero, the CB is motionless according to the following constraint condition. The detection constraint conditions are explained as follows:

- $\left|\overrightarrow{M V_{L 0}}\right|+\left|\overrightarrow{M V_{L 1}}\right|=0$

- $\quad$ Residuals equal to zero. 
However, in spite of the decision process for motionless CBs is efficient, the hit rate of the proposed method should be considered. The probability for the CU are encoded as skip mode under the above two conditions is shown in table 2 . The four average values for each size CU with all four QPs are calculated. Furthermore, as table 2 shows, if the biggest sizes of CUs meet the constraint conditions, it has great opportunity to chose skip mode. Otherwise, the small sizes have low opportunity to be encoded as skip mode. Therefore, this detection process has high efficiency for large size block whereas the small size block has low probability. Moreover, only considering stationary or homogeneous region is incomplete. Due to the considerable numbers of CUs in non-stationary region such as the region of motion region in images could be encoded as skip, the non-stationary regions is necessary to check before the RD optimization process.

Table 2. Probability of skip mode for motionless CB

\begin{tabular}{|l|l|l|l|l|}
\hline \multirow{2}{*}{ sequences } & \multicolumn{5}{|c|}{ Conditional probability with different sizes } \\
\cline { 2 - 5 } & $\mathbf{6 4} \times \mathbf{6 4}$ & $\mathbf{3 2} \times \mathbf{3 2}$ & $\mathbf{1 6} \times \mathbf{1 6}$ & $\mathbf{8} \times \mathbf{8}$ \\
\hline $\begin{array}{l}\text { Traffic } \\
(2560 \times 1600)\end{array}$ & 0.89 & 0.71 & 0.52 & 0.30 \\
\hline $\begin{array}{l}\text { Park Scene } \\
(1920 \times 1080)\end{array}$ & 0.87 & 0.73 & 0.51 & 0.28 \\
\hline $\begin{array}{l}\text { Parry Scene } \\
(832 \times 480)\end{array}$ & 0.93 & 0.70 & 0.47 & 0.26 \\
\hline $\begin{array}{l}\text { Blowing } \\
\text { Bubbles } \\
(416 \times 240)\end{array}$ & 0.87 & 0.69 & 0.55 & 0.27 \\
\hline Average & 0.89 & 0.70 & 0.51 & 0.27 \\
\hline
\end{tabular}

\subsection{Detect the Skip Mode from moving CBs}

In the skip mode coding process, information which is included in the current $\mathrm{CB}$ and corresponding $\mathrm{CBs}$ of the neighbouring video pictures should be the similar, even exactly the same. For this reason, the skip flag and some other associated parameters of referenced CB in previous coded video frames could use directly in the decoder. Furthermore, the mutual information must be very low.

$$
I(C, R)=\sum_{c \in C} \sum_{r \in R} P(c, r) \log _{2}\left[\frac{p(c, r)}{p(c) p(r)}\right]
$$

By utilizing (1), the mutual information $I(c, r)$ of these two current $\mathrm{CB}$ and corresponding $\mathrm{CB}$ could be obtained. The $c$ and $r$ represent the current and corresponding $\mathrm{CBs}$, respectively. And $p(c, r)$ represents the probability function between current and corresponding CBs. However, the function (1) has a large computational complexity since the $p(c, r)$ must compute a two dimensional distribution histogram.

For the maximum reduction computational complexity for function (1), the difference of two entropy values $(\Delta H)$ [21] is introduced in proposed method, which is calculated by (2) and (3) for the two referenced $\mathrm{CU}_{\mathrm{L} 0}$ and $\mathrm{CU}_{\mathrm{L} 1}$ blocks, respectively. And, $H$ represents the entropy that is explained in the (4). Based on (2) and (3), the variation values of entropy between the current $\mathrm{CB}\left(H_{c}\right)$ and the associated $\mathrm{CB}$ of referenced video frames $\left(H_{L 0}\right.$ and $\left.H_{L 1}\right)$ are calculated. Before the calculation process of $\Delta H$ function, (4) should be performed, in which $p(i)$ needs to utilize a one dimensional distribution histogram. First, divide the 256 pixel values. In the proposed fast algorithm, let the set of pixel numbers divide into 26 groups since each group size set as 10 . Then, calculate probability $p(i)$ values. By utilizing the frequency of ten groups luma pixel values, the probability values $p(i)$ is obtained. In the calculation process, it skips probability concepts and replaces with histogram, and this method saves large computational complexity.

$$
\begin{gathered}
\Delta H_{L 0}=a b s\left(H_{L 0}-H_{c}\right) \\
\Delta H_{L 1}=a b s\left(H_{L 1}-H_{c}\right) \\
H_{A}=-\sum_{i \in A} p(i) \log _{2} p(i)
\end{gathered}
$$


The entropy difference values are essential to the proposed method though it exists small errors. Moreover, the difference concept is similar to mutual information method. Thus, when the random numbers $a$ and $b$ are close, the values of $I(a, b)$ and $\Delta H$ with plugging $a$ and $b$ into functions. And, the results would be low and even equal to zero. Furthermore, the coding CU and corresponding CUs are almost the same with aforementioned occasion if they have similar parameters. In general, when a CU is encoded as skip mode, it may have similar entropy with corresponding CU and thus the difference would be equal to zero. Hence, aforementioned entropy difference is same with the occasion of mutual information, which omits the complex calculation process. An experiment has performed to show the accuracy and similarity for these different methods.

Skip mode is not only used in motionless regions but also in the complex texture regions such as the motion regions in texture images. Even though the motion region has an abundant of motion information, the homogeneous CB that should be encoded as skip mode also exists. Generally, the skip mode of a coding block in motion region can reduce the computational complexity obviously. It reveals that the texture features between associated blocks in reference video pictures and current motion blocks are similar. For this reason, the entropy values in skipped block and corresponding two blocks are similar. Moreover, entropy differences must be low since the blocks chose skip mode to code.

The entropy values of non-skipped CB from current and corresponding pictures would be not same since the texture features are different. However, whether the skipped $\mathrm{CB}$ is motionless or not, the texture features are similar to corresponding CB. The entropy difference between current skipped unit and associated coding units is low with negligible error loss.

If the step in computing difference $\Delta H$ was finished, the threshold should be determined for mode decision step in advanced. In our proposed method, decision threshold is self-adaptive. Then, an average difference result for skipped and non-skipped blocks could be obtained by utilizing (5) and (6). The $N_{s}$ and $N_{n s}$ represent some coding blocks have been determined for skipped and non-skipped, respectively. And the parameter $i$ represents the depth of a coding block.

$$
\begin{gathered}
\Delta H_{x_{\text {skip }}}^{i}(t-1)=\left\{\begin{array}{c}
\frac{1}{N_{s}} \sum_{j=0}^{N_{s}-1} \Delta H_{x_{s k i p}}^{i}(j), \text { if } \mathrm{N}_{\mathrm{s}}>0 \\
0, \quad \text { else }
\end{array}\right. \\
\Delta H_{x_{\text {nonskip }}}^{i}(t-1)=\left\{\begin{array}{c}
\frac{1}{N_{n s}} \sum_{j=0}^{N_{n s}-1} \Delta H_{x_{\text {nonskip }}^{i}}^{i}(j), \text { if } \mathrm{N}_{\mathrm{ns}}>0 \\
0, \text { else }
\end{array}\right.
\end{gathered}
$$

The average results $\Delta H$ for skipped or non-skipped coding blocks are affected by time $(t)$. Finally, the distance equations defined here are applied to compute entropy difference of skipped or non-skipped blocks according to above $\Delta H$ results. The functions are as follow:

$$
\begin{gathered}
d_{H_{x}^{s k i p}}=a b s\left\{\Delta H_{x_{s k i p}}^{i}(t)-\Delta H_{x_{\text {skip }}}^{i}(t-1)\right\rfloor \forall \mathrm{x}=L_{0}, L_{1} \\
d_{H_{x}^{\text {nonskip }}}=\operatorname{abs}\left\{\Delta H_{x_{\text {nonskip }}^{i}}^{i}(t)-\Delta H_{x_{\text {nonskip }}}^{i}(t-1)\right\rfloor, \forall \mathrm{x}=L_{0}, L_{1}
\end{gathered}
$$

At last, the constraint condition with threshold for the motion coding blocks are encoded as skip mode:

$$
d_{H_{x}^{\text {skip }}}<d_{H_{x}^{\text {nonskip }}}, \quad \forall \mathrm{x}=L_{0}, L_{1}
$$

On the other hand, others CBs may need further detection procedures for coding as skip or other mode.

\subsection{Overall Algorithm}

1. Start CU encoding decision of a CU block;

2. Calculate the RD cost of the PU mode; 
3. Determinate the skip mode for stationary region based on the conditions of motion vectors which are determined in stationary constraint condition;

4. Perform the check of CUs from list 0 and list 1 ( $C U_{L 0}$ and $C U_{L 1}$ ) of reference frames. If the CU is do not satisfy with stationary constraint condition, go to step 5. Otherwise, the combination of different CBs encoding process skipped;

5. Detect the results derived from step (4) by function (8). If the distance is satisfied with condition (8) when $C U_{L 0}=S K I P$, perform the skipped process. Otherwise, let $C U_{L 1}=S K I P$ and then decide the distance whether satisfied with (8) or not, the CU coding would be skipped if the distance satisfied;

6. Determine skipped CU. Back to step (1) and perform next CU coding detection.

\section{Experimental results}

\subsection{Experimental conditions}

The proposed method is performed on HM10.0 test mode. The QPs values are 22, 27, 32, 37. The frames of video sequences are divided into GOP $=8$ and sequences are tested on the condition of both random access (RA) and low delay (LB) test conditions.

\subsection{The evaluation of proposed method}

The proposed method is performed by utilizing HM test mode 10.0. And, several evaluation criterions of $\triangle B i t, \triangle P S N R$, $\Delta T$, Bjontegaard Delta (BD) [1] rate are used to prove the performance for proposed method. Experimental results are revealed in Table 3. It reveals that our method achieves about $40 \%$ and $39 \%$ coding time reduction under random and low delay conditions while maintains the almost same coding quality compared with original encoder. About 38\%, 44\%, 38\%, and $39 \%$ time reductions of the four groups sequences under random access condition. And, about $44 \%, 34 \%$, 30\%, and $52 \%$ time reductions of the four class sequences under low delay condition. In brief, the early detection method reduces $0.15 \%$ and $0.06 \%$ bit under random and low delay conditions, respectively, with PSNR $0.02 \mathrm{~dB}$ negligible rises. The BD values are $0.43 \%$ and $0.50 \%$ in random and low delay conditions too.

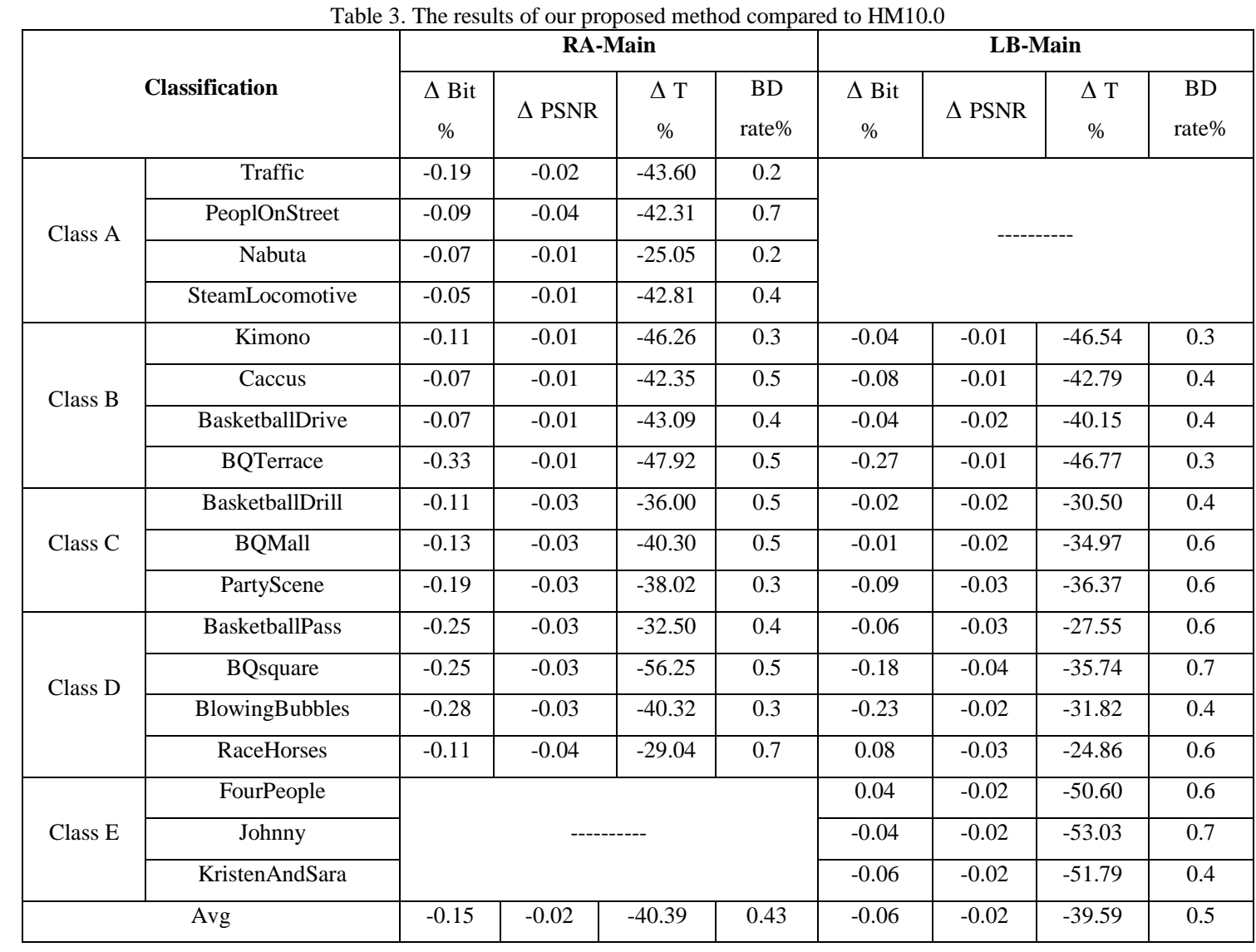


Therefore, the proposed method has a good performance with negligible loss in quality. In addition, as Figure 2 shows, both PSNR and bitrate did not significantly changed for the presented method with respect to original HEVC encoder HM10.0.

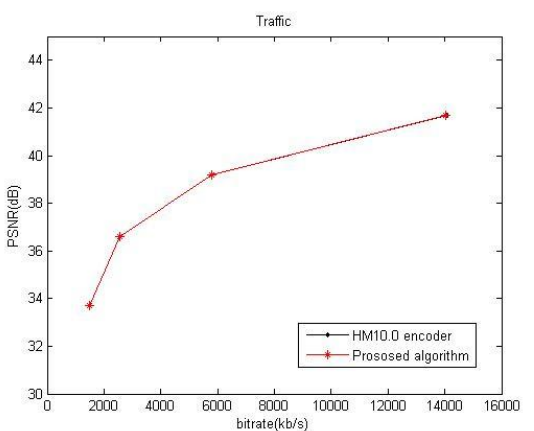

(a)Traffic

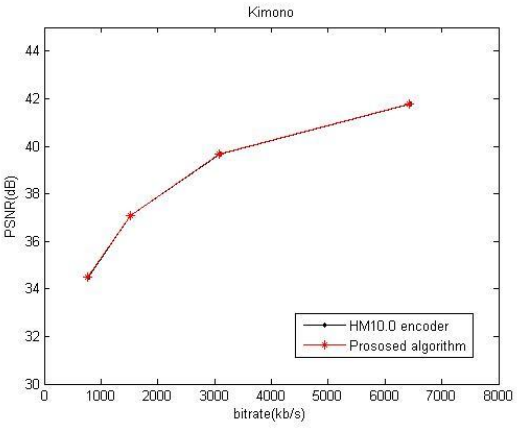

(c)Kimono

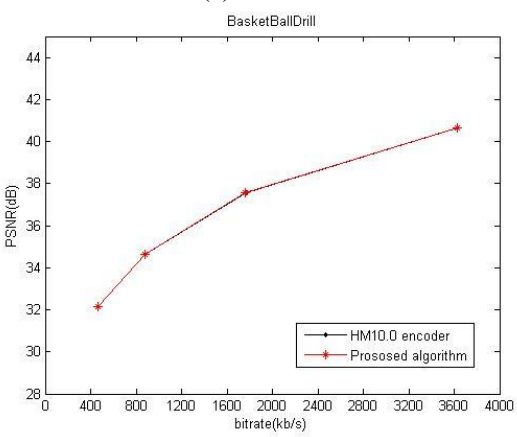

(e)BasketBallDrill

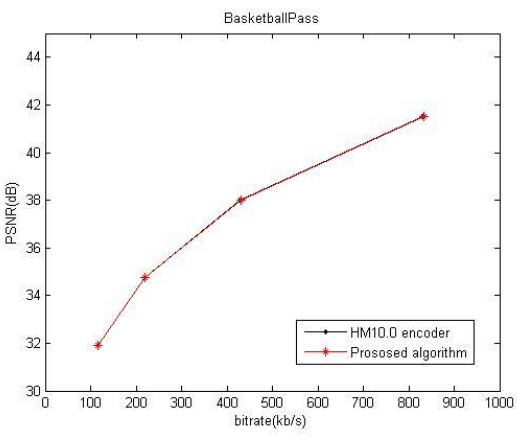

(g)BasketballPass

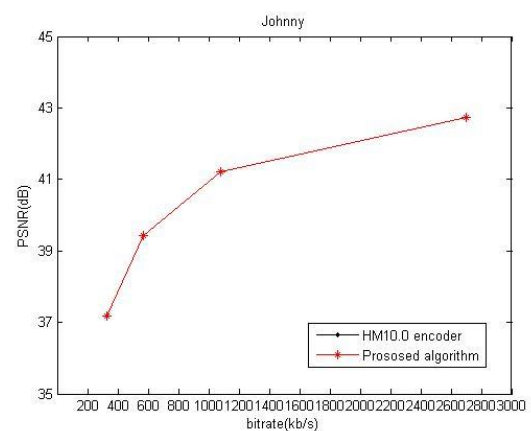

(b)Johnny

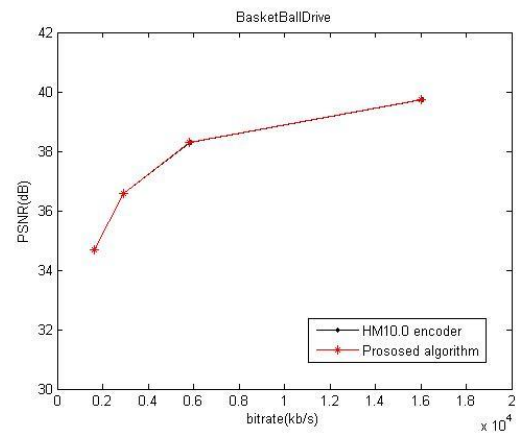

(d)BasketBallDrive

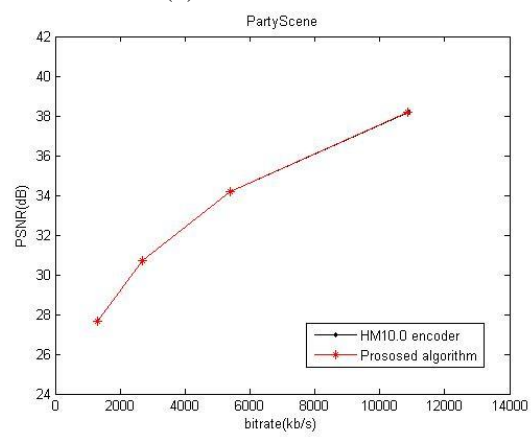

(f)PartyScene

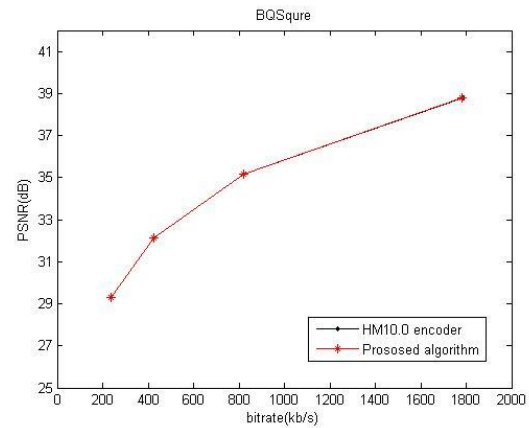

(h)BQSqure

Figure 2. RD curves of (a)“Traffic” (b)“Johnny" (c)“Kimono" (d)“BasketBallDrive” (e)“BasketBallDrill” (f)“PartyScene” (g)“BasketballPass" (h)"BQSqure"

The proposed method also has a better performance of complexity reduction compared to the previous ESD and EPD method under the same condition with this paper. The results are explained in Table 4. Especially, about 10\% time reduction in the condition of random access and low delay compared to ESD. In spite of our presented method result in bit rising contrasts to ESD, the bit reduction achievement compared to conventional HM reference software is apparent. The bit rate 
of proposed method is $0.1 \%$ and $0.09 \%$ higher than ESD from the Table 4. Furthermore, both algorithms implement similar PSNR reduction. The BD of proposed method has about $0.4 \%$ and $0.5 \%$ decrease while ESD realizes about $0.3 \%$ saving in RA and LB condition.

Table 4. The results of our proposed method compared to EPD and ESD

\begin{tabular}{|c|c|c|c|c|c|c|c|c|c|c|c|c|}
\hline \multirow{2}{*}{$\begin{array}{c}\text { avera } \\
\text { ge }\end{array}$} & \multicolumn{4}{|c|}{ EPD } & \multicolumn{4}{|c|}{ ESD } & \multicolumn{4}{|c|}{ Proposed method } \\
\hline & $\begin{array}{c}\Delta \mathrm{Bit} \\
\%\end{array}$ & $\begin{array}{c}\Delta \mathrm{PS} \\
\mathrm{NR}\end{array}$ & $\Delta \mathrm{T} \%$ & $\begin{array}{c}\mathrm{BD} \\
\text { rate } \%\end{array}$ & $\begin{array}{c}\Delta \mathrm{Bit} \\
\%\end{array}$ & $\begin{array}{c}\Delta \mathrm{PS} \\
\mathrm{NR}\end{array}$ & $\Delta \mathrm{T} \%$ & $\begin{array}{c}\mathrm{BD} \\
\text { rate } \%\end{array}$ & $\begin{array}{c}\Delta \text { Bit } \\
\%\end{array}$ & $\begin{array}{c}\Delta \mathrm{PS} \\
\mathrm{NR}\end{array}$ & $\Delta \mathrm{T} \%$ & $\begin{array}{c}\mathrm{BD} \\
\text { rate\% }\end{array}$ \\
\hline $\begin{array}{c}\text { RA- } \\
\text { Main }\end{array}$ & 0.53 & -0.04 & -33.06 & 1.24 & -0.25 & -0.02 & -29.85 & 0.27 & -0.15 & -0.02 & -40.39 & 0.43 \\
\hline $\begin{array}{l}\text { LB- } \\
\text { Main }\end{array}$ & 0.53 & -0.04 & -34.71 & 1.41 & -0.15 & -0.02 & -29.50 & 0.34 & -0.06 & -0.02 & -39.53 & 0.50 \\
\hline
\end{tabular}

The RD cost calculation process of proposed method also implement great time reduction compared with EPD algorithm. In the EPD, set average RD value from skipped CU as decision criterion. If a RD result of $2 N \times 2 N$ coding block lower than given threshold, it would skip to code. The EPD has a time saving about $34.71 \%$, which is less than our proposed method though BD rate is $1.41 \%$.

The ESD only takes into consideration constraint conditions for motionless region, whereas the proposed method considering all motionless and motion regions with better results. Furthermore, previous EPD only utilizes RD values as referenced parameter, whereas the proposed method extra takes into account entropy difference to early decide skipped CU. Hence, this paper proposed a fast algorithm that performs a considering complexity reduction and quality preserve compare with previous method.

Table 5. The results of our proposed method compare with $[13,17,20]$

\begin{tabular}{|c|c|c|c|c|}
\hline $\begin{array}{c}\text { Average } \\
\text { value (\%) }\end{array}$ & Rf [20] & Rf [21] & Rf [22] & $\begin{array}{c}\text { Proposed } \\
\text { algorithm }\end{array}$ \\
\hline $\begin{array}{c}\text { Time } \\
\text { reduction }\end{array}$ & 31.3 & 35.2 & 30 & 39.59 \\
\hline BD & 0.56 & 1.89 & 0.61 & 0.43 \\
\hline
\end{tabular}

Except for the ESD and EPD, the proposed algorithm has a comparison of several algorithms proposed in [13,17,20] next. The results are represented in table 5. The algorithms in Ref. [20] takes advantage of the texture consistency of neighbouring PU in the process of selecting RMD mode and MPM mode. This proposed early intra prediction decision method of HEVC achieves about $31.3 \%$ time saving on average, which is less than proposed algorithm $39.59 \%$ under LB condition with a little higher BD increase about $0.56 \%$ on average. And, the fast inter prediction mode decision method utilizes adaptive threshold in Ref. [17] is proposed based on quantization parameter and mode partition decision. The proposed algorithm with time saving about $39.59 \%$ is better than adaptive threshold method, which saving $35.2 \%$ under LB condition and having better BD rate of $0.43 \%$ compared with $1.89 \%$. The Ref. [13] proposed a technique by exploiting a group of pattern templates (GPTs) utilizing object shapes or edges compared with conventional HEVC block partitioning modes. This technique implies that about $30 \%$ encoding time, which is less than our method about $9.59 \%$ time saving and higher BD growth about $0.18 \%$. The amount of experimental demonstrate that fast coding method utilizing texture analysis of entropy difference is efficient and it consistently outperforms the latest methods of HEVC.

\section{Conclusions}

A fast algorithm for early termination of mode decision in HEVC encoder is proposed in this paper that utilizes entropy method to detect the CBs in the video sequence before the encoding process for skip mode. The texture information is of importance for the detection process of the stationary and non-stationary regions with entropy value is similar to mutual information. The results of comparative experiments reveals that the detection method could apparently reduce the computational complexity about $40 \%$ with respect to HM 10.0 test mode while bring a little loss $(0.43 \%-0.50 \%$ BDBR increase). And the proposed method is better than ESD and EPD algorithm with 8.29\%, 4.39\%, 9.59\% time saving compared with Ref. $[13,17,20]$ and $0.13 \%-1.46 \%$ less BD increased. Experiment results show the proposed method reduces coding time significantly compared to HEVC codec with a negligible quality loss.

\section{Acknowledgements}

The authors would like to thank the editors and anonymous reviewers for their valuable comments. This work was supported in part by the National Natural Science Foundation of China under grant No.61771432, 61302118, 61401404, 
61501407, 61572445, and 61502435, the Program for Science and Technology Innovation Talents in Universities of Henan Province under grant No.17HASTIT022, the Funding Scheme of Young Key Teacher of Henan Province Universities under grant No. 2016GGJS-087, the Scientific and Technological Project of Henan Province under grant No.172102210064, the Scientific and Technological Project of Henan Province under grant No. 142300410248, and 15102210357, the Scientific and Technological of the Education Department of Henan Province under grant No.15A520033, 17B510011, 16A520030, 15A413006, and 16A520028, and in part by the Doctorate Research Funding of Zhengzhou University of Light Industry, under grant No. 2013BSJJ047.

\section{References}

1. G. Bjøntegaard, "Calculation of Average PSNR Differences Between RD-Curves," Proc. 13th VCEG Meeting, Austin, TX, USA, pp. 1-5, 2001

2. Y. Chen, G. Tech, K. Wegner, and S. Yea, “Test Model 11 of 3D-HEVC and MV-HEVC," Joint Collaborative Team on 3D Video Coding Extensions (JCT-3V) document JCT3V-K1003, 11th Meeting: Geneva, CH, 12-18 Feb. 2015

3. K. Goswami, J. H. Lee, and B. G. Kim, "Fast Algorithm for the High Efficiency Video Coding (HEVC) Encoder Using Texture Analysis," Information Sciences, vol. 364, pp. 72-90, Oct. 2016

4. S. Johar, and M. Alwani, "Method for Fast Bits Estimation in Rate Distortion for Intra Coding Units in HEVC," Consumer Communications and Networking Conference (CCNC), Mar. 2013

5. H. S. Kim, and R. H. Park, "Fast CU Partitioning Algorithm for HEVC Using an Online-Learning-Based Bayesian Decision Rule," IEEE Transactions on Circuits and Systems for Video Technology, vol. 26, no. 1, pp. 130-138, Jun. 2015

6. I. K. Kim, J. Min, T. Lee, W. J. Han, and J. H. Park, "Block Partitioning Structure in the HEVC Standard," IEEE Trans. Circuits Syst. Video Technol. Vol. 22, no. 12, pp. 1697-1706, Dec. 2012

7. J. Kim, J. Yang, K. Won, H. Lee, and B. Jeon, "Early SKIP Detection for HEVC," in: Proceedings of JCT-VC, Geneva, Switzerland, Jun. 2012

8. J. H. Lee, K. S. Jang, B. G. Kim, S. Jeong, and J. S. Choi, "Fast Intra Mode Decision Algorithm Based on Local Binary Patterns in High Efficiency Video Coding (HEVC)," Consumer Electronics (ICCE), 2015 IEEE International Conference on, Mar. 2015

9. J. J. Lei, D. Y. Li, Z. Q. Pan, Z. Y. Sun, S. Kwong, and C. Hou, "Fast Intra Prediction Based on Content Property Analysis for Low Complexity HEVC-Based Screen Content Coding," IEEE Transactions on Broadcasting, Vol.63, no. 99, pp. 48-58, Nov. 2016

10. E. G. Mora, J. Jung, M. Cagnazzo, and B. Pesquet, "Initialization, Limitation, and Predictive Coding of the Depth and Texture Quadtree in 3D-HEVC," IEEE Trans. Circuits Syst. Video Technol., vol. 24, no. 9, pp. 1554-1565, Sept. 2014

11. K. Müller, H. Schwarz, D. Marpe, C. Bartnik, S. Bosse, H. Brust, T. Hinz, H. Lakshman, P. Merkle, H. Rhee, G. Tech, M. Winken and T. Wiegand, "3D High Efficiency Video Coding for Multi-view Video and Depth Data," IEEE Trans. Image Process., vol. 22, no. 9, pp. 3366-3378, Sept. 2013

12. J. R. Ohm, G. J. Sullivan, H. Schwarz, T. K. Tan, and T. Wiegand, "Comparison of The Coding Efficiency of Video Coding Standards - Including High Efficiency Video Coding (HEVC),” IEEE Trans. Circuits Syst. Video Technol. Vol. 22, no. 12, 1669-1684, Dec. 2012

13. P. K. Podder, M. Paul, and M. Murshed, “A Novel Depth Edge Prioritization Based Coding Technique to Boost-UP HEVC Performance," Multimedia \& Expo Workshops (ICMEW), 2016 IEEE International Conference on, 26 Sept. 2016

14. M. Radosavljević, G. Georgakarakos, S. Lafond, and D. Vukobratović, "Fast Coding Unit Selection Based on Local Texture Characteristics for HEVC Intra Frame," Signal and Information Processing (Global SIP), 2015 IEEE Global Conference on, Dec. 2015

15. C. Seunghyun, and K. Munchurl, "Fast CU Splitting and Pruning for Suboptimal CU Partitioning in HEVC Intra Coding," IEEE Transactions on Circuits and Systems for Video Technology, Vol. 23, no. 9, pp. 1555-1564, Feb. 2013

16. G. J. Sullivan, J. R. Ohm, W. J. Han, and T. Wiegand, "Overview of the High Efficiency Video Coding (HEVC) Standard," IEEE Trans. Circuits Syst. Video Technol., vol. 22, no. 12, pp. 1649-1668, Dec 2012

17. L. Shen, Z. Zhang, and Z. Liu, "Effective CU Size Decision for HEVC Intra coding," IEEE Trans. Image Processing, vol. 23, no. 10, pp. 4232-4241, Oct. 2014

18. W. Shi, X. T. Jiang, T. Song, and T. Shimamoto, "Edge Information Based Fast Selection Algorithm for Intra Prediction of HEVC," Circuits and Systems (APCCAS), 2014 IEEE Asia Pacific Conference on, Feb. 2015

19. Y. Song, K. Jia, and Q. Wu, "Low Complexity Texture Mode Decision Method for 3D-HEVC," Signal Processing, Communications and Computing (ICSPCC), 2015 IEEE International Conference on, Sept. 2015

20. D. Zhang, Y. Chen, and E. Izquierdo, "Fast Intra Mode Decision for HEVC Based on Texture Characteristic from RMD and MPM," Visual Communications and Image Processing Conference, 2014 IEEE, 02 Mar. 2015

21. H. Zhang, and Z. Ma, "Fast Intra Prediction for High Efficiency Video Coding," in: Advances in Multimedia Information Processing, Springer, pp. 568-577, 2012

22. T. Zhang, M. T. Sun, D. B. Zhao, and W. Gao, "Fast Intra Mode and CU Size Decision for HEVC," IEEE Transactions on Circuits and Systems for Video Technology, Vol. 27, no. 8, PP. 1714-1726, Apr. 2016 